\title{
Dental pulp stem cells express proteins involved in the local invasiveness of odontogenic myxoma
}

\section{Sueli Patricia Harumi Miyagi(a) Carlos Magno da Costa Maranduba ${ }^{(b)}$ Fernando de Sá Silva ${ }^{(c)}$ Márcia Martins Marques ${ }^{(a)}$}

\footnotetext{
(a) Department of Endodontics, School of Dentistry, Universidade de São Paulo, São Paulo, SP, Brazil.

(b) Department of Biology, Institute of Biological Sciences, Universidade Federal de Juiz de Fora, MG, Brazil.

(c) Graduate Program in Biotechnology, Institute of Biomedical Sciences, Universidade de São Paulo, SP, Brazil.
}

\begin{abstract}
Little is known about the histogenesis of the odontogenic myxoma $(\mathrm{OM})$. Dental pulp stem cells could be candidate precursors of OM because both $\mathrm{OM}$ and the dental pulp share the same embryological origin: the dental papilla. For the purpose of comparing OM and stem cells, this study analyzed the expression of two proteins related to OM invasiveness (MMP-2 and hyaluronic acid) in human immature dental pulp stem cells (hIDPSCs). Three lineages of hIDPSCs from deciduous and permanent teeth were used in this study. Immunofluorescence revealed positive reactions for MMP-2 and hyaluronic acid (HA) in all hIDPSCs. MMP-2 appeared as dots throughout the cytoplasm, whereas HA appeared either as diffuse and irregular dots or as short fibrils throughout the cytoplasm and outside the cell bodies. The gene expression profile of each cell lineage was evaluated using RT-PCR analysis, and HA was expressed more intensively than MMP-2. HA expression was similar among the three hIDPSCs lineages, whereas MMP-2 expression was higher in DL-1 than in the other cell lines. The expression of proteins related to OM invasiveness in hIDPSCs could indicate that OM originates from dental pulp stem cells.
\end{abstract}

Descriptors: Stem Cells; Dental Pulp; Myxoma; Odontogenic Tumors.

\section{Introduction}

The odontogenic myxoma $(\mathrm{OM})$ is a benign odontogenic tumor (OT) characterized by local invasiveness and a tendency to recur. ${ }^{1,2}$ Although little is known about the histogenesis of this neoplasm, it is believed to arise from odontogenic ectomesenchyme, since it bears close microscopic resemblance to the mesenchymal portion of a developing tooth, a portion from which the dental pulp also develops. ${ }^{2}$ Dental pulp stem cells could therefore be considered candidate OM precursor cells.

The invasive behavior of OM could be related to the expression of matrix metalloproteinases (MMPs) and extracellular matrix (ECM) components, especially hyaluronic acid (HA). MMPs and HA share the ability of promoting tumor invasion, insofar as HA favors invasion by remodeling the ECM, while MMP-2 facilitates cell proliferation and migration. ${ }^{3-5}$ OM expresses MMP-2 and MMP- $9,{ }^{6}$ as well as high amounts of HA. ${ }^{7}$

MMPs are enzymes collectively capable of degrading all components of the ECM. These enzymes can be divided into five groups: 
- collagenases (MMP-1, MMP-8, MMP-13),

- gelatinases (MMP-2, MMP-9),

- stromelysins (MMP-3, MMP-10, MMP-11),

- membrane-type MMPs (MT-MMP-14, MTMMP-15, MT-MMP-16, MT-MMP-17, MTMMP-23, MT-MMP-24, MT-MMP-25) and

- other novel MMPs. ${ }^{8-10}$

High concentrations of gelatinases are found in invasive tumors, and allow tumor cells to invade normal tissues. ${ }^{11,12}$

The ECM of OM is rich in chondroitin sulfate and HA. ${ }^{13} \mathrm{HA}$ is a ubiquitous and non-sulfated glycosaminoglycan (GAG) with a pseudo-random coil configuration. It measures 2 to $25 \mu \mathrm{m}$ in length, and its molecular weight ranges from 105 to $107 \mathrm{kda} .^{14,15}$ High concentrations of $\mathrm{HA}$ in a tumor prompt the formation of loose, hydrated matrices with a myxoid appearance, capable of facilitating cell division and migration..$^{16,17}$

Our studies have focused on the expression of ECM proteins in human immature dental pulp stem cells (hIDPCSs). ${ }^{18}$ These cells are considered more immature than those studied by Miura et al. ${ }^{19}$ since they are able to express adult and some embryonic stem cell markers. ${ }^{20}$ hIDPSCs are able to express type I collagen, fibronectin and tenascin, ${ }^{18}$ all proteins present in the developing tooth ${ }^{21,22}$ and in $\mathrm{OM} ;{ }^{7}$ however, there are no data on the expression of HA and MMPs in dental pulp stem cells.

One approach to comparing dental pulp stem cells and OM would be to search for the proteins related to OM invasiveness in dental pulp stem cells. Therefore, this study analyzed the distribution and gene expression of MMP-2 and HA in three different lineages of human immature dental pulp stem cells (hIDPSCs).

\section{Methodology}

This study was previously approved by the Research Ethics Committee, University of São Paulo (Protocol \#39/06).

\section{Cell culture}

Three lineages of human immature dental pulp stem cells (hIDPSCs) were used. The hIDPSCs were isolated from the teeth of three different individuals, as follows: two hIDPSC lines were derived from exfoliated human deciduous teeth from a 5- and a 7-year-old child (DL-1 and DL-4, respectively) and the others were obtained from a retained third molar, extracted from a 15-year-old boy (DL-2). DL-1 was obtained from a deciduous tooth with partial root resorption, whereas DL-4 was derived from a deciduous tooth presenting total root resorption. The cells were isolated and characterized as hIDPSCs by Kerkis et al. ${ }^{20}$

Cells grown between the sixth and the tenth passages were used. After thawing the criovials in a $37^{\circ} \mathrm{C}$ water bath for 60 seconds, the cells were cultured in DMEM/Ham's F-12 culture media (1:1, Invitrogen, Carlsbad, USA), supplemented with 15\% fetal bovine serum (FBS, Hyclone, Logan, USA), $100 \mathrm{U} / \mathrm{mL}$ penicillin (Invitrogen), $100 \mu \mathrm{g} / \mathrm{mL}$ streptomycin (Invitrogen), $2 \mathrm{mM}$ L-glutamine (Invitrogen) and $2 \mathrm{mM}$ non-essential amino acids (Invitrogen). ${ }^{20}$ The cells were cultured in an incubator at $37^{\circ} \mathrm{C}$ in humid atmosphere containing $5 \% \mathrm{CO}_{2}$. The cultures were maintained semiconfluent in order to prevent cell differentiation. The cells were passed every 4-5 days by washing the flasks twice in PBS, followed by dissociation in a $0.25 \%$ trypsin solution (Invitrogen). Cell culture media was changed each 2 or 3 days, depending on cell metabolism. For the freezing procedure, cells were re-suspended in a medium containing 20\% FBS, 70\% DMEM, and $10 \%$ dimethylsulfoxide (Sigma, St. Louis, USA) and submitted to lowering temperatures until $-70^{\circ} \mathrm{C}$ was reached. Afterwards, the cells were transferred to liquid nitrogen.

\section{Immunofluorescence protocol}

The cells plated on glass coverslips were fixed with a $4 \%$ paraformaldehyde solution in calciumand magnesium-free phosphate buffered saline (PBSA) for $1 \mathrm{~h}$ and further permeabilized with a $0.1 \%$ Triton X-100 solution in PBSA for $10 \mathrm{~min}$. The cells were then incubated with a $5 \%$ bovine serum albumin (BSA) solution in PBSA for $30 \mathrm{~min}$.

Next, the cells were incubated with the primary antibodies to detect MMP-2 and hyaluronic acid. All antibodies were diluted in a 5\% BSA so- 
lution in PBSA. Optimum antibody concentrations were determined prior to the experiments, using DL-1, DL-2 and DL-4 cells. MMP-2 was detected by a monoclonal mouse antibody from Calbiochem (Calbiochem, Cambrigde, USA), diluted 1:50. Hyaluronic acid was detected by a monoclonal mouse antibody from Biogenesis (Biogenesis, Munich, Germany), diluted 1:50. The secondary antibody was the sheep-anti-mouse antibody conjugated to fluorescein (FITC) from Amersham (Amersham Co., Arlington Heights, USA), diluted in PBSA (1:500) at room temperature. All the incubations were conducted for $60 \mathrm{~min}$ at room temperature, and omission of the primary antibodies served as a negative control.

Observation and photographic recording were carried out under a fluorescence microscope (LSM 410 Zeiss, Oberkochen, Baden-Württemberg, Germany). At least 100 cells were observed for each reaction.

\section{Total RNA extraction and RT-PCR analysis}

Total RNA was extracted from hIDPSCs using the RNeasy Mini kit (Qiagen, Dusseldorf, Germany), according to the manufacturer's instructions. RNA quality was determined by agarose gel electrophoresis and ethidium bromide staining. The $18 \mathrm{~S}$ and 28S RNA bands were visualized under ultraviolet light. The concentration and purity of RNA were determined by measuring absorbance at 260 and $280 \mathrm{~nm}$. Expression of the hyaluronic acid and MMP-2 genes was analyzed on cDNA fragments obtained with the QIAGEN OneStep RT-PCR Kit (Qiagen) using the primers described in Table 1. The products were electrophoresed on $1.5 \%$ agarose gels, stained with ethidium bromide, and visualized with the Gel Logic 100 Imaging System transilluminator (Kodak, New Haven, USA), using Kodak 1D Software, v. 3.6.5 K2 (Kodak).

\section{Results}

The immunofluorescence results and the gene expression reactions are illustrated in Figure 1.

\section{RT-PCR analysis}

Figure 1, top shows the agarose gel images depicting the total RNA bands of the 2 genes analyzed. All hIDPSCs studied expressed MMP-2 and HA genes. The three hIDPSC lines exhibited similar HA gene expression. Total RNA for the MMP-2 gene was expressed markedly in the DL-1 cell line and to a lesser extent in the other two cell lines.

\section{Immunofluorescence}

Positive reactions to both antibodies tested were observed irrespective of the hIDPSC lineage analyzed (Figure 1, bottom). MMP-2 appeared as dots throughout the whole cytoplasm. HA appeared either as diffuse and irregular dots or short fibrils throughout the cytoplasm. Moreover, positive labeling of HA was also observed in the glass coverslip, outside the cell bodies.

\section{Discussion}

The current knowledge of the odontogenic myxoma $(\mathrm{OM})$ still has significant gaps. One of them is related to the histogenesis of this odontogenic neoplasm. A parallel between this neoplasm and dental pulp stem cells was made in an attempt to bring new data to the study of OM histogenesis. This study focused on the expression of proteins related to OM's invasive behavior. The chosen proteins were the matrix metalloproteinase-2 (MMP-2) and hyaluronic acid (HA), since both proteins have been implicated in OM's local invasiveness. ${ }^{6,7}$ As expected, MMP-2 and HA were expressed by the three different lineages of human immature dental pulp stem cells (hIDPSCs) tested. HA gene expression was evident and similar in all hIDPSCs, whereas MMP-2 was expressed markedly in the DL-1 cell line and to a

Table 1 - List of primers used for RT-PCR.

\begin{tabular}{c|c|c|c|c}
\hline Gene & Forward 5' $\rightarrow 3^{\prime}$ & Reverse $5^{\prime} \rightarrow 3^{\prime}$ & Product size (bp) & Annealing \\
\hline MMP-2 & AATGAAGGGACACAGAGGTTTC & CCAGTAGCACCATCATTTCCAC & 198 & 61 \\
\hline Hyaluronic acid & GGTCAGTCCTACAAGATTGGTG & CTTCTCCCAGGCAAGTACAATC & 223 & 61 \\
\hline
\end{tabular}



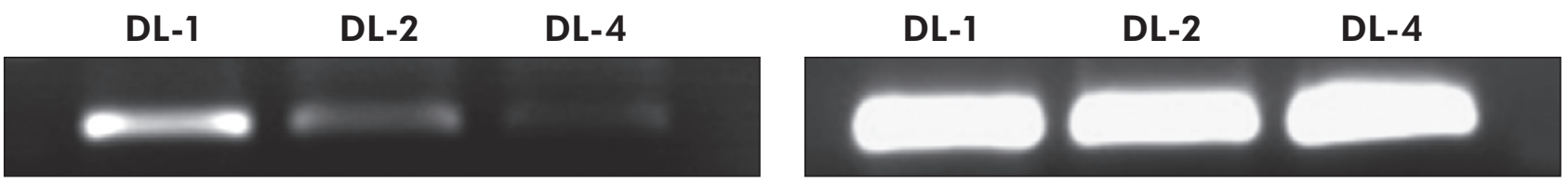

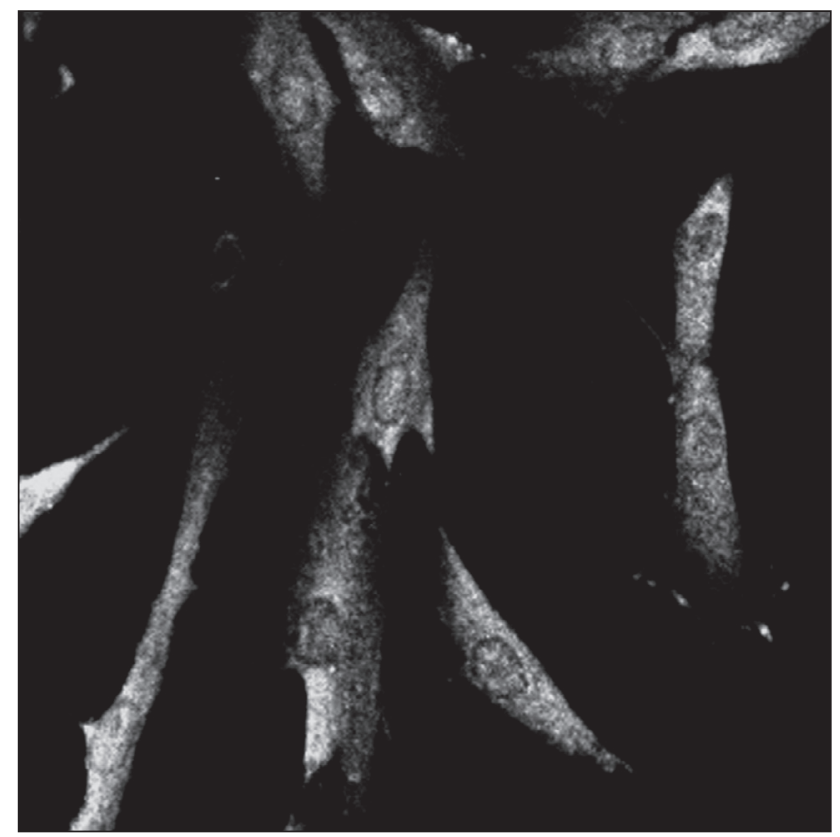

MMP-2

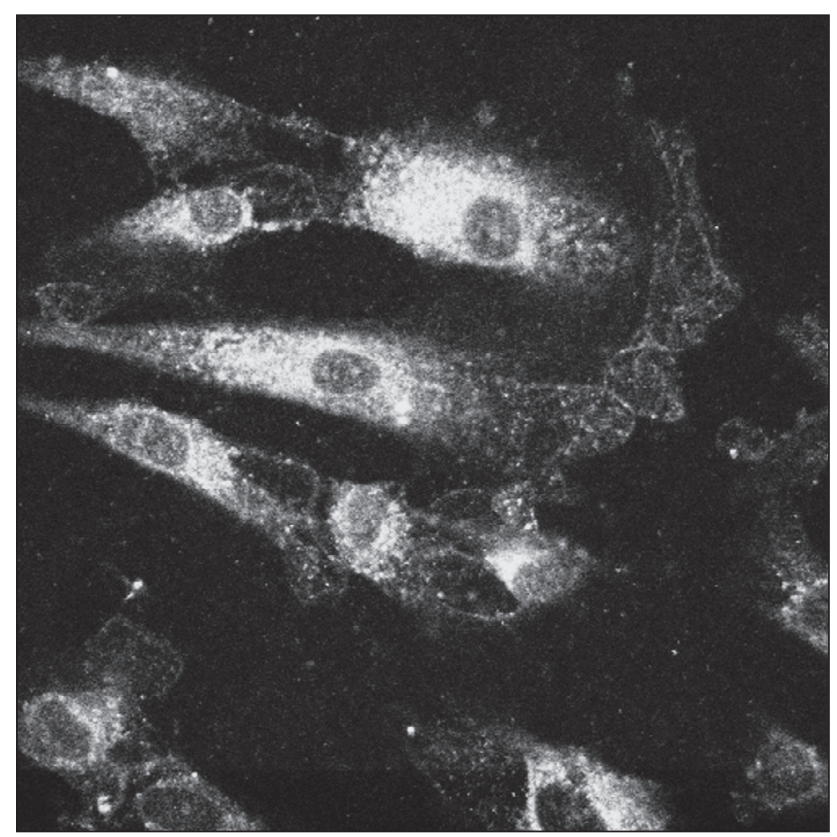

HA

Figure 1 - Representative image of MMP-2 and HA expression: molecular level (gene expression, top) and cellular level (immunofluorescence, bottom). Observe that HA gene expression is similar in all hIDPSCs, whereas the gene expression of MMP-2 varies among the cell lines.

lesser extent in the other two cell lines.

In adult vertebrates, hyaluronic acid is distributed ubiquitously in tissues that require hydrodynamic properties and interactions with other components of the extracellular matrix, such as vitreous synovial fluid and the dermis..$^{14,23-25}$ This protein can also be found in high quantities in inflammation and wound healing processes. ${ }^{14}$ Jaeger et al. ${ }^{7}$ have found high quantities of HA in OM and in a cell line derived from this neoplasm.

High concentrations of HA in embryonic tissues are related to a high rate of proliferation and cell migration. The similarity between the behavior of developing tissues and that of tumors suggests that HA may be a relevant factor for tumor growth..$^{3-5}$ In fact, high levels of HA may stimulate tumor progression. ${ }^{26,27}$ As an example of this HA activity, stromal levels of HA have been linked to a worse prognosis in cases of ovarian carcinoma. ${ }^{28}$
The exuberance of this ECM protein in OM observed in vivo and in vitro strongly suggests that it has a definitive role in the local invasiveness of this neoplasm. Thus, it would be expected that a precursor cell of OM should be capable of producing this protein. All hIDPSCs expressed HA at the molecular and cellular levels. These results - along with the previous observation of other ECM molecules (e.g., type I collagen, fibronectin and tenascin) expressed by these cells and present in the tumor ${ }^{18}-$ are indicative of at least a strong resemblance between hIDPSCs and OM cells.

Matrix metalloproteinases (MMPs) are enzymes capable of degrading components of the extracellular matrix $(\mathrm{ECM}){ }^{8,9}$ High concentrations of these enzymes can be found in more invasive tumors, enabling tumor cells to invade normal tissues. ${ }^{11,12} \mathrm{Mi}$ yagi et al. ${ }^{6}$ have found a high expression of MMP-2 in a cell line derived from an OM. These authors 
have chosen to search for MMP-2 because this gelatinase is frequently involved in the invasive phenotype of some lesions. This characteristic is based on its ability to degrade type IV collagen. ${ }^{29}$ Other authors have also found a high expression of MMP2 in OM. Bast et al. ${ }^{30}$ reported MMP-2 expression in $90 \%$ of the OM cases studied. Although all hIDPSCs expressed MMP-2 in our study, the level of expression was dependent on the hIDPSC lineage considered. Based on this parallel between OM and dental pulp stem cells, the positive reactions observed at the molecular and cellular levels corroborate the hypothesis that dental pulp stem cells could be precursors of OM. However, the observed differences in expression warrant further investigation.

In a previous study, we observed differences in the expression of ECM proteins among different hIDPSCs. ${ }^{18}$ These differences were observed in the expression of type I collagen and were explained based on the donor tooth conditions (deciduous or permanent, retained or erupted, and degree of root resorption). ${ }^{18}$ The DL-2 cells derived from a retained permanent tooth had the lowest expression of type I collagen, whereas the DL-1 and DL-4 cells derived from erupted teeth had a significantly higher expression. ${ }^{18}$ In this study the highest expression of MMP2 was observed in DL-1, followed by DL-2, and the lowest was observed in DL-4. The expression of this enzyme declines with donor tooth aging. In fact, the DL- 4 cell line was derived from a deciduous tooth at an advanced stage of root resorption, whereas DL-1, although also derived from a deciduous tooth, was

\section{References}

1. Sumi Y, Miyaishi O, Ito K, Ueda M. Magnetic resonance of myxoma in the mandible: a case report. Oral Surg Oral Med Oral Pathol Oral Radiol Endod. 2000 Nov;90(5):671-6.

2. Barros RE, Dominguez FV, Cabrini RL. Myxoma of the jaws. Oral Surg Oral Med Oral Pathol. 1969 Feb;27(2):225-36.

3. Knudson W, Biswas C, Li XQ, Nemec RE, Toole BP. The role and regulation of tumour-associated hyaluronan. Ciba Found Symp. 1989;143:150-9.

4. Camenisch TD, Spicer AP, Brehm-Gibson T, Biesterfeldt J, Augustine ML, Calabro A Jr, et al. Disruption of hyaluronan synthase-2 abrogates normal cardiac morphogenesis and at an early stage of root resorption. On the other hand, MMP-2 expression was intermediate in DL-2 cells, although the donor tooth from which this cell line was derived had a non traumatized dental pulp, and was also an older tooth. Based on these observations, it may be assumed that MMP-2 expression could also be related to donor tooth conditions.

\section{Conclusion}

The expression of MMP-2 and HA observed in all the hIDPSCs studied reveals an interesting resemblance between OM cells and dental pulp stem cells. These results, combined with those of a previous study showing other ECM proteins expressed by $\mathrm{OM}$ also found in hIDPSCs, ${ }^{18}$ strongly suggest that dental pulp stem cells can be the precursors of OM. New studies either searching for other similarities between this neoplasm and dental pulp stem cells, or seeking to reproduce the neoplasm by inducing hIDPSCs, among other research possibilities, may contribute to clarifying the histogenesis of odontogenic tumors.

\section{Acknowledgments}

This study was supported by grants from FAPESP (Fundação de Amparo à Pesquisa do Estado de São Paulo, Brazil; Grants 06/50296-7). We are grateful to Dr. Irina Kerkis from the Genetic Laboratory, Instituto Butantan, São Paulo, SP, Brazil, for kindly providing us with human immature dental pulp stem cells. We also thank Ms. Cícera Maria Gomes for helping us with the molecular biology technique.

hyaluronan-mediated transformation of epithelium to mesenchyme. J Clin Invest. 2000 Aug;106(3):349-60.

5. Toole BP, Hascall VC. Hyaluronan and tumor growth. Am J Pathol. 2002 Sep;161(3):745-7.

6. Miyagi SP, Hiraki KR, Martins MD, Marques MM. Expression of matrix metalloproteinases 2 and 9 in odontogenic myxoma in vivo and in vitro. J Oral Sci. 2008 Jun;50(2):18792.

7. Jaeger MMM, Santos J, Domingues M, Ruano R, Araújo N, Caroli A, et al. A novel cell line retains the morphological characteristics of the cells and matrix of odontogenic myxoma. J Oral Pathol Med. 2000 Mar;29(3):129-38. 
8. Parks WC. Matrix metalloproteinases in repair. Wound Repair Regen. 1999 Nov-Dec;7(6):423-32.

9. Overall CM, López-Otín C. Strategies for MMP inhibition in cancer: innovations for the post-trial era. Nat Rev Cancer. 2002 Sep;2(9):657-72.

10. Demers M, Couillard J, Bélanger S, St-Pierre Y. New roles for matrix metalloproteinases in metastasis. Crit Rev Immunol. 2005;25(6):493-523.

11. Curran S, Murray GI. Matrix metalloproteinases in tumour invasion and metastasis. J Pathol. 1999 Nov;189(3):300-8.

12. Coussens LM, Fingleton B, Matrisian LM. Matrix metalloproteinase inhibitors and cancer: trials and tribulations. Science. 2002 Mar;295(5564):2387-92.

13. Barker BF. Odontogenic myxoma. Semin Diagn Pathol. 1999 Nov;16(4):297-301.

14. Toole BP, Slomiany MG. Hyaluronan: a constitutive regulator of chemoresistance and malignancy in cancer cells. Semin Cancer Biol. 2008 Aug;18(4):244-50.

15. Gaffney J, Matou-Nasri S, Grau-Olivares M, Slevin M. Therapeutic applications of hyaluronan. Mol Biosyst. 2010 Mar;6(3):437-43.

16. Day AJ, Prestwich GD. Hyaluronan-binding proteins: tying up the giant. J Biol Chem. 2002 Feb 15;277(7):4585-8.

17. Stern R. Devising a pathway for hyaluronan catabolism: are we there yet? Glycobiology. 2003 Dec;13(12):105R-115R.

18. Harumi Miyagi SP, Kerkis I, da Costa Maranduba CM, Gomes CM, Martins MD, Marques MM. Expression of extracellular matrix proteins in human dental pulp stem cells depends on the donor tooth conditions. J Endod. 2010 May;36(5):82631.

19. Miura M, Gronthos S, Zhao M, Lu B, Fisher LW, Robey PG, et al. SHED: stem cells from human exfoliated deciduous teeth. Proc Natl Acad Sci U S A. 2003 May 13;100(10):5807-12.

20. Kerkis I, Kerkis A, Dozortsev D, Stukart-Parsons GC, Gomes Massironi SM, Pereira LV, et al. Isolation and characterization of a population of immature dental pulp stem cells expressing
OCT-4 and other embryonic stem cell markers. Cells Tissues Organs. 2006;184(3-4):105-16.

21. Garcia JM, Martins MD, Jaeger RG, Marques MM. Immunolocalization of bone extracellular matrix proteins (type I collagen, osteonectin and bone sialoprotein) in human dental pulp and cultured pulp cells. Int Endod J. 2003 Jun;36(6):40410.

22. Abrahão IJ, Martins MD, Katayama E, Antoniazzi JH, Segmentilli A, Marques MM. Collagen analysis in human tooth germ papillae. Braz Dent J. 2006;17(3):208-12.

23. Toole BP. Hyaluronan in morphogenesis. Semin Cell Dev Biol. 2001 Apr;12(2):79-87.

24. Toole BP. Hyaluronan: from extracellular glue to pericellular cue. Nat Rev Cancer. 2004 Jul;4(7):528-39.

25. Jiang D, Liang J, Noble PW. Hyaluronan in tissue injury and repair. Annu Rev Cell Dev Biol. 2007;23:435-61.

26. Enegd B, King JA, Stylli S, Paradiso L, Kaye AH, Novak U. Overexpression of hyaluronan synthase- 2 reduces the tumorigenic potential of glioma cells lacking hyaluronidase activity. Neurosurgery. 2002 Jun;50(6):1311-8.

27. Wang SJ, Bourguignon LY. Role of hyaluronan-mediated CD44 signaling in head and neck squamous cell carcinoma progression and chemoresistance. Am J Pathol. 2011 Mar;178(3):956-63.

28. Anttila MA, Tammi RH, Tammi MI, Syrjänen KJ, Saarikoski SV, Kosma VM. High levels of stromal hyaluronan predict poor disease outcome in epithelial ovarian cancer. Cancer Res. 2000 Jan;60(1):150-5.

29. Aresu L, Benali S, Garbisa S, Gallo E, Castagnaro M. Matrix metalloproteinases and their role in the renal epithelial mesenchymal transition. Histol Histopathol. 2011 Mar;26(3):30713.

30. Bast BT, Pogrel MA, Regezi JA. The expression of apoptotic proteins and matrix metalloproteinases in odontogenic myxomas. J Oral Maxillofac Surg. 2003 Dec;61(12):1463-6. 